\title{
Does my brain want what my eyes like? - How food liking and choice influence spatio-temporal brain dynamics of food viewing
}

\author{
Marie-Laure Bielser ${ }^{\star a}$, Camille Crézé ${ }^{\star b}$, Micah M. Murray ${ }^{\mathrm{a}, \mathrm{c}, \mathrm{d}}$, Ulrike Toepel ${ }^{\mathrm{a}}$ \\ a The Laboratory for Investigative Neurophysiology (The LINE), Department of Radiology and \\ Department of Clinical Neurosciences, Vaudois University Hospital Center and University of Lausanne, \\ Lausanne, Switzerland \\ b Department of Physiology, University of Lausanne, Lausanne, Switzerland \\ c Electroencephalography Brain Mapping Core, Center for Biomedical Imaging (CIBM) of Lausanne \\ and Geneva, Switzerland \\ d Department of Hearing and Speech Sciences, Vanderbilt University, Nashville, TN 37232, USA \\ * equal contribution
}

Correspondence address:

Dr. Ulrike Toepel

Laboratory for Investigative Neurophysiology (LINE)

Departments of Clinical Neurosciences and Radiology

Centre Hospitalier Universitaire Vaudois and University of Lausanne

Hôpital Nestlé, 5 av. Pierre Decker

1011 Lausanne, Switzerland

Tel: $0041-213141317$

E-mail: Ulrike.toepel@unil.ch

Keywords: EEG, VEP, food, valuation, choice 


\section{Abstract}

How food valuation and decision-making influence the perception of food is of major interest to better understand food intake behavior and, by extension, body weight management. Our study investigated behavioral responses and spatio-temporal brain dynamics by means of visual evoked potentials (VEPs) in twenty-two normal-weight participants when viewing pairs of food photographs. Participants rated how much they liked each food item (valuation) and subsequently chose between the two alternative food images.

Unsurprisingly, strongly liked foods were also chosen most often. Foods were rated faster as strongly liked than as mildly liked or disliked irrespective of whether they were subsequently chosen over an alternative. Moreover, strongly liked foods were subsequently also chosen faster than the less liked alternatives. Response times during valuation and choice were positively correlated, but only when foods were liked; the faster participants rated foods as strongly liked, the faster they were in choosing the food item over an alternative.

VEP modulations by the level of liking attributed as well as the subsequent choice were found as early as $135-180 \mathrm{~ms}$ after food image onset. Analyses of neural source activity patterns over this time interval revealed an interaction between liking and the subsequent choice within the insula, dorsal frontal and superior parietal regions. The neural responses to food viewing were found to be modulated by the attributed level of liking only when foods were chosen, not when they were dismissed for an alternative. Therein, the responses to disliked foods were generally greater than those to foods that were liked more. Moreover, the responses to disliked but chosen foods were greater than responses to disliked foods which were subsequently dismissed for an alternative offer. Our findings show that the spatio-temporal brain dynamics to food viewing are immediately influenced both by how much foods are liked and by choices taken on them. These valuation and choice processes are subserved by brain regions involved 
in salience and reward attribution as well as in decision-making processes, which are likely to influence prospective dietary choices in everyday life. 


\section{Introduction}

A better understanding of how humans evaluate foods and make choices about them, particularly if associated with objective brain markers underlying decision-making processes, are of great interest because eating-related disorders and especially obesity figures are still increasing world-wide. In daily life, decisions on what to eat are determined by hunger (homeostatic needs), and also by hedonic drives that can even override homeostatic needs (Berthoud, 2011; Kenny, 2011). The latter have been strongly associated with the propensity of food indulgence, leading to overweight and detrimental health consequences like cardiovascular disease and diabetes. In light of such developments, it is critical to better understand how food choice decisions are shaped as well as the underlying neural mechanisms, with the long-term goal to potentially being able to affect maladaptive choices in favor of health-beneficial ones.

A core concept in research on human economic and food-related decisions states that when several choice options are present at the same time, they are assigned abstract and often subjective values. These values, and especially between-value weighing, serves to enable decisions in favor of a (more highly valued) choice option (Economides et al., 2015; Hare et al., 2009; Kable and Glimcher, 2009). Several functional neuroimaging studies have revealed that in particular the ventromedial prefrontal cortex (vmPFC) subserves the computation of an overall subjective value of choice options and thereby biases decisions (Kable and Glimcher, 2009). Dorsal and ventrolateral PFC (dIPFC and vIPFC, respectively) have been shown to modulate vmPFC activity and are involved in inhibitory control processes (Hare et al., 2009; Hutcherson et al., 2012) as well as emotional self-regulation (Ochsner and Gross, 2008). For example, activity of the vmPFC was found to be modulated by lateral PFC activity in individuals who are successful (self-) controllers of their dietary choices (Hare et al., 2009). In the study of Hare and colleagues (2009), the success of self-control on dietary choices (meaning that study participants successfully considered the health aspects of food in their dietary choices) was 
positively associated with the activity in the dIPFC, subsequently influencing food value attribution as reflected by vmPFC modulations. Other neuroimaging studies have further shown that the brain tracks the energy content of foods (García-García et al., 2013; Killgore et al., 2003; Van der Laan et al., 2011). Responses to varying food types (Toepel et al., 2009) and food portion sizes (Toepel et al., 2015) have been shown to differ already within $200 \mathrm{~ms}$ after image exposure; reflected by activity modulations in temporo-occipital and frontal brain regions (e.g. orbitofrontal cortex, anterior cingulate cortex, lateral prefrontal cortex, and the insula).

To date, only few neuroimaging studies have investigated modulations in brain responses during food viewing according to valuation or liking, respectively, including the impact of valuation on subsequent food choices. In nutrition sciences and research on human food choice behavior, a prominent concept posits a dissociation of processes related to food 'liking' as opposed to 'wanting', as well as how 'liking' and 'wanting' impact food choices and intake (Berridge, 2009). Consequently, foods that are not necessarily 'liked' can nevertheless trigger implicit 'wanting' mechanisms (e.g. when adequate alternatives are lacking) and thus impact food choices and intake (Berridge, 2009; Finlayson et al., 2007). By contrast, 'liking' is not necessarily coupled with 'wanting' since this would be maladaptive for dietary choices given the abundance of foods with strong hedonic impact in everyday life.

Our study aimed to determine how food liking and successive choices shape the spatiotemporal brain dynamics during food image viewing. Visual evoked potentials (VEPs) were analyzed within an electrical neuroimaging framework (Murray et al., 2008; Koenig et al., 2014). This approach not only includes the analysis of VEPs at the head-surface, but also intracranial source estimations. We have successfully employed this method when investigating uni- and multisensory responses to food (Lietti et al., 2012; Ohla et al., 2012; Toepel et al., 2009, 2012, 2014, 2015). We hypothesized that the level of liking attributed to foods directly impacts VEPs 
and neural source activity during food viewing. Moreover, we questioned whether participants' successive choices (i.e. whether food items were subsequently chosen or dismissed for an alternative offer) would differentially impact brain responses during food viewing, given that choices between alternatives only had to be taken subsequent to, but not during, food viewing.

\section{Materials and Methods}

\section{Participants in the EEG study}

Twenty-two (11 female) normal-weighted volunteers, aged 21-38 yrs (mean \pm s.e.m. = $27.23 \pm 0.98$ yrs; mean BMI \pm s.e.m. $\left.=22.72 \pm 0.61 \mathrm{~kg} / \mathrm{m}^{2}\right)$, participated in the study. Twenty of these participants were right-handed, and two were ambidextrous according to the Edinburgh Handedness Inventory (Oldfield, 1971). None of the participants reported current or prior neurological or psychiatric illnesses or self-reported eating disorders, and all participants had normal or corrected-to-normal vision. All participants completed the Three-Factor-Eating questionnaire TFEQ-R 18 (Karlsson et al., 2000) and the momentary craving state questionnaire (FCQ-S) (Nijs et al., 2007). The EEG recording sessions started between 15:00 and 16:00h to control for circadian modulations of hunger. Furthermore, participants were instructed (and also themselves reported) to have eaten lunch $\sim 2 \mathrm{~h}$ before the recording sessions. The volunteers provided written, informed consent to the procedures, which were approved by the Ethics Committee of the Faculty of Biology and Medicine of the University of Lausanne and the Vaudois University Hospital Center (CHUV).

\section{Procedure of the EEG study}

Participants sat comfortably inside a dimly lit, sound-attenuated booth and completed 704 trials (structure as schematized in Figure 1). Images were presented on a 21" CRT monitor. 
On each trial, participants were successively presented with two food items and rated each during a 'valuation phase' on a 5-point Likert according to how much they liked each food item presented (Literal translation from French: $1=$ The item does not seem pleasant to me.; $5=$ The item seems very pleasant to me.). Following each image pair, participants were presented with a question mark indicating the 'choice period'. They were told to decide by button press whether they preferred the first (button press 1) or the second food (button press 2) viewed. All behavioral responses were given by button-presses on a serial response box using the index finger. Responses were allowed during the presentation of the food image and 2000ms after image/ question mark offset. Participants were instructed not to respond at all in case they encountered unknown food items. Stimulus presentation and response recordings were controlled by E-Prime (Psychology Software Tools Inc., Pittsburgh, USA; www.pstnet.com/eprime).

Image pairs consisted of foods from a similar product category (e.g. milk products, meat products, desserts, salty snacks, fruits, vegetables) and were adapted in energy content (Toepel et al., 2009) to prevent effects from general preferences for e.g. only sweet or high-energy foods. Each food image pair was presented twice to each participant with inversed image order to avoid position effects of foods within a trial. Images were controlled for low-level visual features, including luminance and spatial frequency (Knebel et al., 2008).

\section{- $\quad$ Insert Figure 1 about here -}

\section{Behavioral data analyses}

First, we analyzed participants' behavioral responses to each food item given during the valuation phase. By using customized MATLAB scripts, individuals' responses to each food were sorted by the level of liking participants had attributed, as well as by whether the food 
items were subsequently "chosen" vs. "dismissed". Since extreme ratings were low in number, food items scored with "1" and "2" were pooled together, as well as those rated with " 4 " and " 5 ". That is, overall six conditions entered the analyses, i.e. chosen food items that had been rated as rather disliked, as mildly liked and rather strongly liked, as well as dismissed food items which had been rated as rather disliked, as mildly liked and strongly liked. For each condition, the frequency of responses and reaction times were first averaged within and then across subjects. 2x3 ANOVAs with the factors of choice (chosen vs. dismissed) and liking (rather disliked, mildly and strongly liked) were conducted on response frequencies as well as response times. When appropriate, separate one-way ANOVAs for each choice option as well as paired post-hoc t-tests (two-tailed) were conducted.

Second, we analyzed participants' response times to the chosen food item during the 'choice period' of each trial. Therein, responses to chosen foods were (this time retrospectively) sorted depending on whether the respective food item had been rated as rather disliked, mildly liked or strongly liked. A one-way ANOVA including the three liking levels was conducted to investigate liking-related differences in food choices, as well as paired post-hoc t-tests when appropriate.

Third, we conducted Pearson correlation analyses (two-tailed) between individuals' response times in the valuation and choice period to investigate whether decisions about food liking (as assessed by response times) are predictive of food choice decisions. The outcomes of all performed analyses were only considered significant when $p \leq 0.05$.

\section{Electroencephalography (EEG) acquisition and preprocessing}

Continuous EEG was acquired at $512 \mathrm{~Hz}$ through a 128-channel Biosemi ActiveTwo system (Biosemi, Amsterdam, The Netherlands) referenced to a ground circuitry (common mode sense and driven right leg electrodes or CMS-DRL). This circuitry functions as a feedback loop driving the average potential across the montage as close as possible to the 
amplifier zero. Details of this circuitry, including a diagram, can be found on the Biosemi website (http://www.biosemi.com/pics/zero ref1 big.gif). All data pre-processing steps and VEP averaging were done using the Cartool software (http://sites.google.com/site/fbmlab/cartool; (Brunet et al., 2011). All statistical analyses were conducted using the Statistical Toolbox for Electrical Neuroimaging (STEN) developed by Jean-François Knebel (http://www.unil.ch/line/home/menuinst/about-the-line/software--analysis-tools.html).

To calculate VEPs, epochs of EEG from $98 \mathrm{~ms}$ pre- to $488 \mathrm{~ms}$ post-food image onset (i.e. 50 data points before and 250 data points after stimulus onset) were separately averaged for each response condition and each participant. In addition to an automatic $\pm 80 \mu \mathrm{V}$ artifact rejection criterion, EEG epochs containing eye blinks or other noise transients were removed by trial-to-trial inspection of the data. Data were high-pass and low-pass filtered during singlesubject averaging (second order Butterworth with -12db/octave roll-off; $0.1 \mathrm{~Hz}$ high-pass; $40 \mathrm{~Hz}$ low-pass; $50 \mathrm{~Hz}$ notch) and baseline corrected using the $98 \mathrm{~ms}$ pre-stimulus period. Data from artifact electrodes of each participant were interpolated using 3-D splines (Perrin et al., 1987), and single-subject EEG epochs were averaged into VEPs depending on the behavioral response of each participant on each trial (see above). EEG responses were thus sorted into six conditions, i.e. responses to (subsequently) chosen food that had been rated as rather disliked, as mildly liked and strongly liked, as well as responses to (subsequently) dismissed foods that had been rated as rather disliked, as mildly liked and rather strongly liked. Single subject's responses were then group-averaged and recalculated against the average reference. The average number of accepted VEP epochs ranged from 60 (s.e.m. \pm 12 ) to 175 (s.e.m. \pm 16 ) per condition. Due to the differing number of epochs per condition, VEP epochs were normalized by the mean instantaneous Global Field Power (GFP) at each sampling point (Lehmann and Skrandies, 1980) during group averaging. GFP is calculated as the square root of the mean of the squared amplitude value recorded at each electrode of the 128-channel montage we used 
(vs. the average reference) and represents the spatial standard deviation of the electric field measured on the head surface (Koenig and Melie-Garcia, 2010).

\section{Analyses of VEP data}

The influence of liking and choices on brain responses was quantified by assessments of modulations in the VEPs at the head-surface and in estimations of the underlying neural activity (Brunet et al., 2011; Murray et al., 2008). Periods of interest were determined by a timepoint and electrode-wise 2x3 ANOVA with the factors of choice (chosen vs. dismissed) and liking (disliked, mildly and strongly liked). Only periods showing a significant interaction of liking $x$ choice $(p \leq 0.05)$ for longer than 20 ms on more than $10 \%$ of all channels were considered significant, so as to account for temporal and spatial auto-correlation (e.g. Guthrie and Buchwald, 1991).

In parallel, we assessed the electric field strength at the scalp surface for each response condition, viz. Global Field Power (GFP; (Lehmann and Skrandies, 1980) in the VEP responses. GFP is calculated as the square root of the mean of the squared amplitude value recorded at each electrode of the 128-channel montage (vs. the average reference) and represents the spatial standard deviation of the electric field at the scalp. That is, GFP yields larger amplitudes for stronger electric fields, and GFP peaks are indicative of maximally synchronized neural sources underlying the scalp-recorded activity (Michel and Murray, 2012). Over the periods of interest (i.e. showing an interaction between the factors of choice and liking at the VEP level), mean GFP values were computed for individuals and used for statistical analyses. First, $2 \times 3$ ANOVAs with the factors of choice (chosen vs. dismissed) and liking (rather disliked, mildly and strongly liked) were conducted. When appropriate, separate one-way ANOVAs for each choice option condition as well as t-tests (two-tailed) were conducted to detail the impact of each level of liking attributed on subsequent food choice. All these results were only considered significant when $p \leq 0.05$. 
To assess whether and which neural sources (i.e. brain regions) revealed activity patterns modulated by liking and choices, we then estimated the active sources over the GFP maxima in each condition using the local autoregressive average (LAURA) distributed linear inverse solution (Grave de Peralta Menendez et al., 2001, 2004). As input for these estimations, single subject VEP responses at each electrode were averaged over the (overlapping) interval of the GFP maxima and electrode-wise VEP differences, by this generating a single value for each participant and each response condition to increase the signal to noise ratio. The LAURA algorithm then serves to estimate the neural sources of the electric signal recorded at the 128 head-surface sensors by using an inverse solution matrix consisting of 3005 nodes equally distributed within the grey matter of the Montreal Neurological Institute (MNI) average brain. This implementation of LAURA was generated with the Spherical Model with Anatomical Constraints (SMAC; (Spinelli et al., 2000). As output, LAURA provides current density values (in $\mathrm{mA} / \mathrm{mm}^{3}$ ) at each node. The spatial accuracy attained, which is on the order of the grid size (here: 6x6x6mm; (Gonzalez Andino et al., 2005a, 2005b; Michel et al., 2004), has been documented and discussed in detail in prior fundamental and clinical research.

Modulations in neural source activity over each GFP maximum were assessed by means of $2 \times 3$ ANOVAs on each node of the solution point matrix with the factors of choice (chosen vs. dismissed) and liking (rather disliked, mildly and strongly liked). Activity in brain regions where a significant interaction of both factors was found served as regions of interest for post-hoc analyses. These regions of interest were considered significant when the statistical threshold of $p \leq 0.05$ (two-tailed) was exceeded within a cluster of $\geq 10$ contiguous nodes of the inverse solution matrix. This spatial extension criterion was based on AlphaSim randomizations (http://afni.nimh.nih.gov) and also used in previous publications of our group (Lietti et al., 2012; Toepel et al., 2009, 2012, 2014). Post-hoc comparisons (separate one-way ANOVAs for responses to chosen and dismissed foods as well as t-tests between) were conducted on the averaged scalar values (in $\mu \mathrm{A} / \mathrm{mm}^{3}$ ) of the node revealing the minimal $p$-value within a region of 
interest plus its six immediate neighbors. The results analyses were rendered on the MNI template brain with the Talairach and Tournoux (1988) coordinates of the maximal statistical differences indicated.

\section{Results}

\section{Behavioral results}

Figure $2 \mathrm{a}$ shows the mean response frequencies (in percent) to chosen and dismissed food items as a function of the liking level attributed. A 2x3 ANOVA revealed an interaction of liking and choice $\left(F_{2,42}=46.93 ; p \leq 0.01 ; \eta_{p}^{2}=0.69\right)$ indicating that the number of food items rated as rather disliked, mildly or strongly liked substantially differed depending on whether they were subsequently chosen over an alternative or dismissed. Separate one-way ANOVAs for each choice condition revealed an effect of liking for chosen $\left(F_{2,42}=17,88 ; p \leq 0.01 ; \eta_{p}{ }^{2}=0.46\right)$, but not for dismissed food items. Within the chosen foods items, paired t-tests showed that strongly liked ones were preferred more often over an alternative than mildly liked $\left(t_{21}=3.57 ; p \leq 0.01\right)$ and disliked ones $\left(t_{21}=4.54 ; p \leq 0.01\right)$. Also, food items rated as mildly liked were chosen more often than disliked ones $\left(t_{21}=2.09 ; p \leq 0.05\right)$. Paired t-tests between the two choice options (chosen vs. dismissed) revealed that rather disliked foods were chosen less often than dismissed $\left(t_{21}=6.30\right.$; $\mathrm{p} \leq 0.01)$, but that strongly liked foods were indeed more often chosen than dismissed $\left(\mathrm{t}_{21}=8.12\right.$; $\mathrm{p} \leq 0.01$ ). Figure $2 \mathrm{~b}$ displays mean reaction times of participants (in milliseconds) to food items as a function of how much they were liked. A $2 \times 3$ ANOVA with the factors of choice (chosen vs. dismissed food items) and liking (rather disliked, mildly or strongly liked) only revealed a main effect of liking $\left(F_{2,42}=307.62 ; p \leq 0.01 ; \eta_{p}{ }^{2}=0.94\right)$. For post-hoc comparisons, responses were thus collapsed across choices (chosen and dismissed). Paired t-tests between liking levels 
showed that food items rated as strongly liked were rated faster than mildly liked $\left(t_{21}=25.08\right.$; $\mathrm{p} \leq 0.01)$ and disliked ones $\left(\mathrm{t}_{21}=14.20 ; \mathrm{p} \leq 0.01\right)$.

Figure 2c illustrates reaction times of participants over the period of choice between the two presented food alternatives in each trial. A one-way ANOVA revealed a main effect of liking $\left(F_{2,42}=5.883 ; p \leq 0.01 ; \eta_{p}{ }^{2}=0.22\right)$. Post-hoc paired t-tests between liking levels revealed that participants were faster when choosing food images that had previously been rated as strongly liked as compared to mildly liked $\left(t_{21}=2.26 ; p \leq 0.05\right)$ and rather disliked $\left(t_{21}=3.25 ; p \leq 0.01\right)$. Figure $2 d$ illustrates the associations between participants' response times in the food valuation phase and the subsequent food choice period. When participants were fast in rating foods as mildly or strongly liked, they were likewise faster in making a decision in favor of these foods over the choice period (mildly liked foods: $r_{20}=0.46 ; p \leq 0.05$; strongly liked foods: $r_{20}=0.54 ; p \leq 0.01$ ). The supplementary table provides a comprehensive numerical overview of participants' responses given during the valuation phase and the food choice period.

- $\quad$ Insert Figure 2 about here -

\section{Modulations of head-surface responses to food viewing by liking and choices}

The electrode-wise $2 \times 3$ ANOVA on the head-surface VEPs over time revealed an interaction between liking and subsequent choice over the $135-180 \mathrm{~ms}$ post-image onset interval (Figure 3a). The effect was particularly prominent at frontal electrodes, though we would remind the reader that analyses of voltage waveforms are dependent on the choice of the reference electrode, including also when the average reference is used. We therefore also analyzed a reference-independent measure - GFP - taking a mean value over the time window identified above (i.e. 135-180ms; bar graph in Figure 3b). The 2x3 ANOVA conducted revealed an

interaction of liking and choice $\left(F_{2,20}=4.84 ; p \leq 0.05 ; \eta_{p}^{2}=0.33\right)$. Separate one-way ANOVAs for 
each choice condition (chosen and dismissed) revealed that the GFP was only modulated by the level of liking attributed when later chosen foods were viewed $\left(F_{2,20}=3.72 ; p \leq 0.05 ; \eta_{p}{ }^{2}=\right.$ $0.27)$, but not when later dismissed foods were viewed $\left(p=0.09 ; \eta_{p}{ }^{2}=0.21\right)$. Within chosen foods, post-hoc t-tests then showed that the electric field strength when viewing strongly liked foods was higher than when mildly liked foods were viewed $\left(t_{21}=2.78 ; p \leq 0.01\right)$. Paired $t$-tests between responses to equally liked chosen and dismissed foods showed that the GFP was higher for successively chosen strongly liked foods as opposed to their dismissed counterparts $\left(t_{21}=3.19 ; p \leq 0.01\right){ }^{1}$

- Insert Figure 3 about here -

\section{Modulations in neural source activity to food viewing by liking and choice}

A whole-head 2x3 ANOVA with the factors of liking (rather disliked, mildly and strongly liked) and choice (chosen vs. dismissed) served to define regions of interest for post-hoc contrasts (Figure 4). An interaction of the factors liking and choice (visualized in Figure 3a) was observed in the right dorsolateral PFC (dIPFC; Max: $57,-5,35$ ), the insula of the left hemisphere (INS; Max: $x=-34, y=-11, z=14$ ), and in the right superior parietal cortex (SPC; Max: $x=22, y=-$ $40, z=65)$. Neural activity in these regions was modulated by whether foods are liked and whether they are successively chosen or dismissed for an alternative.

Separate one-way ANOVAs on neural activity in the abovementioned areas to foods that were either chosen or dismissed revealed modulations only when subsequently chosen foods were viewed, but not when later dismissed foods had been encountered (dIPFC: $F_{2,42}=3.25$; $p=0.05 ;$ INS: $F_{2,42}=5.42 ; p \leq 0.01 ;$ SPC: $\left.F_{2,42}=3.28 ; p \leq 0.05\right)$. Post-hoc t-tests on the neural activity

\footnotetext{
${ }^{1}$ Note: We did not find correlations between participants' behavioral data, anthropometric characteristics or food intake attitudes with the GFP responses.
} 
pattern in these regions showed the following results. In the dIPFC, the difference in neural activity was most pronounced when subsequently chosen mildly liked foods were viewed as opposed to their dismissed counterparts $\left(t_{21}=2.67 ; p \leq 0.05\right)$. Moreover, the activity when viewing mildly liked subsequently chosen foods was higher than when strongly liked later chosen foods were viewed $\left(t_{21}=2.49 ; p \leq 0.05\right)$. In the insula, neural activity when viewing rather disliked foods was higher when they were subsequently chosen as when they were later dismissed for an alternative $\left(t_{21}=3.00 ; p \leq 0.01\right)$, and also greater as when mildly liked foods were viewed that were later chosen $\left(t_{21}=3.29 ; p \leq 0.01\right)$. In the superior parietal region, neural activity was found greater when rather disliked foods were viewed that were subsequently chosen as compared to when disliked foods were later dismissed for an alternative $\left(t_{21}=3.71 ; p \leq 0.01\right)$. Activity was also greater when comparing the neural responses to disliked subsequently chosen foods and strongly liked chosen foods $\left(t_{21}=2.48 ; p \leq 0.05\right)$.

The whole-head 2x3 ANOVA we conducted additionally revealed a main effect of liking (visualized in Figure 4b) in the dorsomedial prefrontal region (Max: $x=-20, y=30, z=42$ ) indicating that this region's responsiveness is modulated by food liking rather independent of whether foods are subsequently chosen or dismissed for an alternative. For post-hoc comparisons, data points for chosen and dismissed foods were thus collapsed, and revealed stronger activity to the viewing of rather disliked as opposed to mildly $\left(t_{21}=2.33 ; p \leq 0.05\right)$ and strongly liked foods $\left(t_{21}=2.17 ; p \leq 0.05\right)$.

Moreover, the whole-head 2x3 ANOVA showed an additional main effect of choice (visualized in Figure 4c) in the right dorsolateral prefrontal cortex (Max: $x=27, y=49, z=20$ ) indicating that activity in this region is in particular modulated by food choices, independent of how much the viewed foods were liked. For post-hoc comparisons, data points were thus 
collapsed across liking levels, and revealed stronger activity to the viewing of subsequently chosen as opposed to dismissed foods $\left(t_{21}=2.37 ; p \leq 0.05\right) .^{2}$

- Insert Figure 4 about here -

\section{Discussion}

Our study identified the impact of food liking and successive choices on the spatio-temporal brain dynamics during food viewing in normal-weight participants. Behaviorally, our results showed (unsurprisingly) that strongly liked food items were more frequently chosen than dismissed, and that disliked items were more frequently dismissed than chosen. Nonetheless and regardless of the subsequent choice (i.e. whether food items were chosen or dismissed), participants were faster in rating food items as strongly liked (vs. disliked or mildly liked). Moreover, they were faster in making a choice in favor of foods that had been rated as strongly liked as opposed to mildly liked and disliked ones. Response times in both behavioral tasks, food valuation and food choice, were positively correlated when foods had been rated as mildly or strongly liked, showing that a fast food valuation response goes along with a fast decision in favor of a food item, but only when foods are liked. In other words, liking a food facilitates its choice over an alternative, but our study design cannot elucidate whether a similar relation in reaction times is apparent when foods are disliked and consecutively dismissed for an alternative.

These findings are in line with previous literature reporting decreased response times to highly valued visual items (Kahnt et al., 2014). Given that in our study liked items were also

\footnotetext{
${ }^{2}$ Note: We did not find correlations between participants' behavioral data, anthropometric characteristics or food intake attitudes with the neural source estimation measures.
} 
more often chosen by participants in general, this association is likely due to the inherent parallel assessment of 'liking' and 'wanting' aspects during preference building (Finlayson et al., 2008). We would nonetheless note that the food choice task we employed in our study is not appropriate to investigate deliberate 'wanting' (Berridge, 2009) and to thus enable assertions on a clear dissociation of 'liking' and 'wanting' processes. Participants were not given the opportunity of 'free' choices between food options, but they had to perform forced decisions between two alternatives. We will further refer to this point in the discussion on elevated brain responses to disliked foods that were still chosen over an alternative.

With respect to spatio-temporal brain dynamics, VEP modulations during food viewing as a function of liking and subsequent choice were apparent within the first $150 \mathrm{~ms}$ after image onset. The timing of these modulations converges with previously reported effects of food categorization in terms of energy content (Toepel et al., 2009) and effects of judging meal portion sizes for expected satiety (Toepel et al., 2015). Estimations of neural source activity over the time interval from $135-180$ ms showed an interaction of liking and choice in the neural activity patterns of the dorsolateral PFC, the insula and the superior parietal lobe. Only brain responses to foods that were successively chosen were modulated by the level of liking, whereas no modulation was observed for responses to foods that were later dismissed for an alternative. Within the responses to chosen foods, strongest neural activity was often associated with the viewing of disliked foods that were nevertheless preferred over an alternative. Moreover, we identified two brain areas whose activity was either modulated by food liking only, independent of whether the viewed foods were subsequently chosen or dismissed for an alternative (i.e. dorsomedial PFC), or was altered by choice only, independent of how much the viewed foods had been rated as liked (i.e. lateral PFC).

Modulations in neural activity during food viewing by the level of liking attributed and subsequent choices thus involved a network of regions associated with salience-related attentional and cognitive control processes (Menon and Uddin, 2010; Mitchell, 2011). Harris and 
colleagues (2013) showed that the dIPFC is involved in the early top-down modulation of attention when participants had to perform a decision-making task and exercise self-control while viewing appetizing food images. This electrical neuroimaging study reported higher neural activity over the time interval between 150-200ms when participants successfully chose healthy foods for prospective consumption while dismissing unhealthy food alternatives. These effects temporally converge with the time interval over which we observed pronounced modulations of neural activity by the level of liking attributed to foods that were successively chosen over an alternative. Therein, the viewing of disliked foods that were nevertheless chosen over an alternative elicited the greatest activity.

The prefrontal cortex has consistently been found to be involved in decision-making and emotion regulation in different experimental paradigms and modalities (Mitchell, 2011; Shenhav and Buckner, 2014). Whereas the dorsomedial PFC is involved in the encoding of rewardrelated information, i.e. stimulus values, in the context of decision-making (Camus et al., 2009), the dorsolateral prefrontal cortex is particularly recruited when choice options have to be weighed and self-control has to be exerted (Hare et al., 2009; Kober et al., 2010; McClure et al., 2007). When facing conflicts between choice options, the activity of the dmPFC and dIPFC often increases in parallel, allowing for an adjustment of cognitive control and rendering of most relevant stimulus features as salient in order to guide choice behavior (Egner and Hirsch, 2005; Mitchell, 2011; Mitchell et al., 2009; Walton et al., 2007). Directly related to decisions on food options, increased dIPFC activity was reported in successful rather than in unsuccessful dieters (Hare et al., 2009).

Insula modulations, on the other hand, have been associated with task-related signaling in the context of food valuation (Born et al., 2011). In particular middle-to-posterior insular regions are involved in the exertion of self-control over food intake (Harris et al., 2013) and the integration of interoceptive sensations (Craig, 2002). Due to its high connectivity with the dIPFC and the vmPFC (Carmichael and Price, 1996; Craig, 2002), the insula is thought to be a key 
player enabling interactions between regions for stimulus valuation and choice (Harris et al., 2013). Prior electrical neuroimaging studies have shown that insular activity is elevated within 150ms when participants view meal portion sizes judged as inappropriate for prospective intake (Toepel et al., 2015) further indicating its role in the integration of homeostatic and hedonic information.

The superior parietal cortex (SPC), another region showing modulations by liking and choices in our data, has been ascribed a role in attentional processes (Bisley and Goldberg, 2010), e.g. when non-food objects are viewed (Levy et al., 2011). Moreover, the superior parietal cortex was also found to be involved in the abstract coding of stimulus values in order to mediate goal-directed behaviors (Kahnt et al., 2014) and decisions between choice alternatives (Kable and Glimcher, 2009; McClure et al., 2007). Electrical neuroimaging results have shown SPC modulations within $200 \mathrm{~ms}$ when visual cues require an abstract value coding to enable decisions between choice options (Harris et al., 2011).

In our study, both the insula and the posterior parietal region showed elevated responses especially when viewing foods rated as disliked, but subsequently chosen; likely since the alternative food option was not considered more tempting. That is, an increased stimulus valuation mediating decisions between two choice options cannot account for the obtained results. An alternative explanation of the observed patterns might be a particularly strong choice conflict when both food alternatives presented in one experimental trial were rated as rather disliked, but a choice had to nonetheless be indicated despite of the low liking level attributed to both images. In order to address this question, we computed indices of choice coherence in individuals (Supplementary figure), and also investigated whether individuals' choice coherence was associated with neural source activity.

First, we calculated for each participant the \%response to foods that were chosen among the two alternatives presented per experimental trial and had been attributed the highest level of liking (coherent choice). In addition, individuals' \%response to foods chosen although 
not attributed the highest level of liking in the respective experimental trials were computed (incoherent choice). Moreover, neutral choices were defined as instances were both food items had been attributed the identical level of liking. In a next step, the percentage of incoherent choices was subtracted from the percentage of coherent choices in each participant and for each level of liking to obtain 'choice coherence indices'. A one-way ANOVA on participants' choice coherence including the three levels of liking (disliked, mildly liked and strongly liked) revealed an effect of liking. For highly liked foods, choices were more coherent than for mildly liked than for disliked foods. However, choice coherence was not correlated with neural source activity during the viewing of disliked but chosen food items in any of the previously discussed brain regions. The additional analysis thus could not provide evidence for a relation between a strong choice conflict (assumed for disliked yet chosen foods) and elevated neural responses when foods were viewed that were rated as disliked, yet subsequently chosen over an alternative option. We would like to point out that we attempted further analyses (i.e. reaction times as a function of choice coherence; impact of difference in liking levels attributed to food alternative 1 and 2 per trial on our findings), but it should become clear from the supplementary figure that there were only very few data points for some conditions hindering these efforts. That is, we cannot fully exclude the possibility of a strong choice conflict for disliked food items induced by the forced-choice task in our design.

However, in a recent EEG study top-down attentional influences on food viewing responses were found within $200 \mathrm{~ms}$ when controlled decisions need to be taken (Harris et al. 2013). In this study, participants viewed appetizing food products under varying tasks. In one part of the experiment, participants were asked to make random food choices without constraints. In a second part they had to exert self-control during food viewing with the goal to receive monetary incentives for longitudinal body weight loss. Over the $150-200 \mathrm{~ms}$ interval during food viewing, successful self-control trials (which were defined as accepting healthy, but disliked foods or rejecting unhealthy, but liked foods) elicited stronger activity in the insula, the 
dorsolateral PFC and the ventrolateral PFC. Harris and colleagues proposed that this result pattern is due to attentional filtering mechanisms serving to mediate self-control when needed, i.e. in order to adjust food choice behavior.

Although our study design differs from the one of Harris et al. (2013) in task instructions (food choices without vs. with exertion of self-control to support body weight management) and visual food 'valuation conditions' (self-attributed liking vs. taste/health ratings), results of both studies converge on the timing of early response modulations, and partially on the brain network involved. That is, we have reason to believe that in our study responses to foods rated as disliked, but not ultimately chosen, are influenced by attentional modulations of brain regions involved in salience attribution and decision-making. Notably, our study design does not permit the comparison of responses to disliked-chosen with strongly liked-rejected food items to further strengthen parallels with the study of Harris et al. (2013). Moreover, valuation and choice were highly intertwined in our study as they are in everyday dietary behavior, and future research still needs to disentangle the influences of valuation and choice on the spatio-temporal brain dynamics to food viewing. Yet, further investigations of choice conflicts between food alternatives (similar or differing in attributes like perceived pleasantness, health impact and tastiness) are certainly needed to complete insights in human food intake behavior and their role in body weight management and its health consequences.

\section{Acknowledgments}

The Cartool software used for EEG data analysis has been programmed by Denis Brunet (Functional Brain Mapping Laboratory, Geneva, Switzerland) and is supported by the Center for Biomedical Imaging (CIBM) of Geneva and Lausanne. Ulrike Toepel is supported by an Interdisciplinary Research Grant from the Faculty of Biology and Medicine, University of 
Lausanne, Switzerland. Micah Murray is supported by the Swiss National Science Foundation (grant \#320030-149982) and the Swiss Brain League (2014 Research Prize). 


\section{References}

Berridge, K.C. (2009). "Liking" and "wanting" food rewards: brain substrates and roles in eating disorders. Physiol. Behav. 97, 537-550.

Berthoud, H.-R. (2011). Metabolic and hedonic drives in the neural control of appetite: who is the boss? Curr. Opin. Neurobiol. 21, 888-896.

Bisley, J.W., and Goldberg, M.E. (2010). Attention, intention, and priority in the parietal lobe. Annu. Rev. Neurosci. 33, 1-21.

Born, J.M., Lemmens, S.G.T., Martens, M.J.I., Formisano, E., Goebel, R., and WesterterpPlantenga, M.S. (2011). Differences between liking and wanting signals in the human brain and relations with cognitive dietary restraint and body mass index. Am. J. Clin. Nutr. 94, 392-403.

Brunet, D., Murray, M.M., and Michel, C.M. (2011). Spatiotemporal analysis of multichannel EEG: CARTOOL. Comput. Intell. Neurosci. 2011, 813870.

Camus, M., Halelamien, N., Plassmann, H., Shimojo, S., O'Doherty, J., Camerer, C., and Rangel, A. (2009). Repetitive transcranial magnetic stimulation over the right dorsolateral prefrontal cortex decreases valuations during food choices. Eur. J. Neurosci. 30, 1980-1988.

Carmichael, S.T., and Price, J.L. (1996). Connectional networks within the orbital and medial prefrontal cortex of macaque monkeys. J. Comp. Neurol. 371, 179-207.

Craig, A.D. (2002). How do you feel? Interoception: the sense of the physiological condition of the body. Nat. Rev. Neurosci. 3, 655-666. 
Economides, M., Guitart-Masip, M., Kurth-Nelson, Z., and Dolan, R.J. (2015). Arbitration between controlled and impulsive choices. Neurolmage 109, 206-216.

Egner, T., and Hirsch, J. (2005). Cognitive control mechanisms resolve conflict through cortical amplification of task-relevant information. Nat. Neurosci. 8, 1784-1790.

Finlayson, G., King, N., and Blundell, J.E. (2007). Is it possible to dissociate "liking" and "wanting" for foods in humans? A novel experimental procedure. Physiol. Behav. 90, 36-42.

Finlayson, G., King, N., and Blundell, J. (2008). The role of implicit wanting in relation to explicit liking and wanting for food: implications for appetite control. Appetite 50, 120-127.

García-García, I., Narberhaus, A., Marqués-Iturria, I., Garolera, M., Rădoi, A., Segura, B., Pueyo, R., Ariza, M., and Jurado, M.A. (2013). Neural responses to visual food cues: insights from functional magnetic resonance imaging. Eur. Eat. Disord. Rev. J. Eat. Disord. Assoc. 21, 89-98.

Gonzalez Andino, S.L., Murray, M.M., Foxe, J.J., and de Peralta Menendez, R.G. (2005a). How single-trial electrical neuroimaging contributes to multisensory research. Exp. Brain Res. 166, 298-304.

Gonzalez Andino, S.L., Michel, C.M., Thut, G., Landis, T., and Grave de Peralta, R. (2005b). Prediction of response speed by anticipatory high-frequency (gamma band) oscillations in the human brain. Hum. Brain Mapp. 24, 50-58.

Grave de Peralta Menendez, R., Gonzalez Andino, S., Lantz, G., Michel, C.M., and Landis, T. (2001). Noninvasive localization of electromagnetic epileptic activity. I. Method descriptions and simulations. Brain Topogr. 14, 131-137. 
Grave de Peralta Menendez, R., Murray, M.M., Michel, C.M., Martuzzi, R., and Gonzalez Andino, S.L. (2004). Electrical neuroimaging based on biophysical constraints. Neurolmage 21, $527-539$.

Guthrie, D., Buchwald, J.S. (1991). Significance testing of difference potentials.

Psychophysiology 28, 240-244.

Hare, T.A., Camerer, C.F., and Rangel, A. (2009). Self-control in decision-making involves modulation of the vmPFC valuation system. Science 324, 646-648.

Harris, A., Adolphs, R., Camerer, C., and Rangel, A. (2011). Dynamic construction of stimulus values in the ventromedial prefrontal cortex. PloS One 6, e21074.

Harris, A., Hare, T., and Rangel, A. (2013). Temporally dissociable mechanisms of self-control: early attentional filtering versus late value modulation. J. Neurosci. Off. J. Soc. Neurosci. 33, 18917-18931.

Hutcherson, C.A., Plassmann, H., Gross, J.J., and Rangel, A. (2012). Cognitive regulation during decision making shifts behavioral control between ventromedial and dorsolateral prefrontal value systems. J. Neurosci. Off. J. Soc. Neurosci. 32, 13543-13554.

Kable, J.W., and Glimcher, P.W. (2009). The Neurobiology of Decision: Consensus and Controversy. Neuron 63, 733-745.

Kahnt, T., Park, S.Q., Haynes, J.-D., and Tobler, P.N. (2014). Disentangling neural representations of value and salience in the human brain. Proc. Natl. Acad. Sci. U. S. A. 111, 5000-5005. 
Karlsson, J., Persson, L.O., Sjöström, L., and Sullivan, M. (2000). Psychometric properties and factor structure of the Three-Factor Eating Questionnaire (TFEQ) in obese men and women. Results from the Swedish Obese Subjects (SOS) study. Int. J. Obes. Relat. Metab. Disord. J. Int. Assoc. Study Obes. 24, 1715-1725.

Kenny, P.J. (2011). Reward Mechanisms in Obesity: New Insights and Future Directions. Neuron 69, 664-679.

Killgore, W.D.S., Young, A.D., Femia, L.A., Bogorodzki, P., Rogowska, J., and Yurgelun-Todd, D.A. (2003). Cortical and limbic activation during viewing of high- versus low-calorie foods. Neurolmage 19, 1381-1394.

Knebel, J.-F., Toepel, U., Hudry, J., le Coutre, J., and Murray, M.M. (2008). Generating controlled image sets in cognitive neuroscience research. Brain Topogr. 20, 284-289.

Kober, H., Mende-Siedlecki, P., Kross, E.F., Weber, J., Mischel, W., Hart, C.L., and Ochsner, K.N. (2010). Prefrontal-striatal pathway underlies cognitive regulation of craving. Proc. Natl. Acad. Sci. U. S. A. 107, 14811-14816.

Koenig, T., Stein, M., Grieder, M., and Kottlow, M. (2014). A tutorial on data-driven methods for statistically assessing ERP topographies. Brain Topogr 27, 72-83.

Koenig, T., Melie-Garcia, L.A. (2010.) A Method to Determine the Presence of Averaged EventRelated Fields Using Randomization Tests. Brain Topogr 23, 233-242.

Lehmann, D., and Skrandies, W. (1980). Reference-free identification of components of checkerboard-evoked multichannel potential fields. Electroencephalogr. Clin. Neurophysiol. 48, 609-621. 
Levy, I., Lazzaro, S.C., Rutledge, R.B., and Glimcher, P.W. (2011). Choice from non-choice: predicting consumer preferences from blood oxygenation level-dependent signals obtained during passive viewing. J. Neurosci. Off. J. Soc. Neurosci. 31, 118-125.

Lietti, C.V., Murray, M.M., Hudry, J., le Coutre, J., and Toepel, U. (2012). The role of energetic value in dynamic brain response adaptation during repeated food image viewing. Appetite 58 , $11-18$.

McClure, S.M., Ericson, K.M., Laibson, D.I., Loewenstein, G., and Cohen, J.D. (2007). Time discounting for primary rewards. J. Neurosci. Off. J. Soc. Neurosci. 27, 5796-5804.

Menon, V., and Uddin, L.Q. (2010). Saliency, switching, attention and control: a network model of insula function. Brain Struct. Funct. 214, 655-667.

Michel, C.M., and Murray, M.M. (2012). Towards the utilization of EEG as a brain imaging tool. Neurolmage 61, 371-385.

Michel, C.M., Murray, M.M., Lantz, G., Gonzalez, S., Spinelli, L., and Grave de Peralta, R. (2004). EEG source imaging. Clin. Neurophysiol. Off. J. Int. Fed. Clin. Neurophysiol. 115, 21952222.

Mitchell, D.G.V. (2011). The nexus between decision making and emotion regulation: a review of convergent neurocognitive substrates. Behav. Brain Res. 217, 215-231.

Mitchell, D.G.V., Luo, Q., Avny, S.B., Kasprzycki, T., Gupta, K., Chen, G., Finger, E.C., and Blair, R.J.R. (2009). Adapting to dynamic stimulus-response values: differential contributions of inferior frontal, dorsomedial, and dorsolateral regions of prefrontal cortex to decision making. J. Neurosci. Off. J. Soc. Neurosci. 29, 10827-10834. 
Murray, M.M., Brunet, D., and Michel, C.M. (2008). Topographic ERP analyses: a step-by-step tutorial review. Brain Topogr. 20, 249-264.

Nijs, I.M.T., Franken, I.H.A., and Muris, P. (2007). The modified Trait and State Food-Cravings Questionnaires: development and validation of a general index of food craving. Appetite 49, 3846.

Ochsner, K.N., and Gross, J.J. (2008). Cognitive Emotion Regulation: Insights from Social Cognitive and Affective Neuroscience. Curr. Dir. Psychol. Sci. 17, 153-158.

Ohla, K., Toepel, U., le Coutre, J., and Hudry, J. (2012). Visual-gustatory interaction: orbitofrontal and insular cortices mediate the effect of high-calorie visual food cues on taste pleasantness. PloS One 7, e32434.

Oldfield, R.C. (1971). The assessment and analysis of handedness: the Edinburgh inventory. Neuropsychologia 9, 97-113.

Perrin, F., Pernier, J., Bertrand, O., Giard, M.H., and Echallier, J.F. (1987). Mapping of scalp potentials by surface spline interpolation. Electroencephalogr. Clin. Neurophysiol. 66, 75-81.

Shenhav, A., and Buckner, R.L. (2014). Neural correlates of dueling affective reactions to winwin choices. Proc. Natl. Acad. Sci. U. S. A. 111, 10978-10983.

Spinelli, L., Andino, S.G., Lantz, G., Seeck, M., and Michel, C.M. (2000). Electromagnetic inverse solutions in anatomically constrained spherical head models. Brain Topogr. 13, 115125.

Talairach, J., Tournoux, P. (1988). Co-planar Stereotaxic Atlas of the Human Brain: 3Dimensional Proportional System - an Approach to Cerebral Imaging. New York: Thieme Medical Publishers. 
Toepel, U., Knebel, J.-F., Hudry, J., le Coutre, J., and Murray, M.M. (2009). The brain tracks the energetic value in food images. Neurolmage 44, 967-974.

Toepel, U., Knebel, J.-F., Hudry, J., le Coutre, J., and Murray, M.M. (2012). Gender and weight shape brain dynamics during food viewing. PloS One 7, e36778.

Toepel, U., Ohla, K., Hudry, J., le Coutre, J., and Murray, M.M. (2014). Verbal labels selectively bias brain responses to high-energy foods. Neurolmage 87, 154-163.

Toepel, U., Bielser, M.-L., Forde, C., Martin, N., Voirin, A., le Coutre, J., Murray, M.M., and Hudry, J. (2015). Brain dynamics of meal size selection in humans. Neurolmage 113,133-142. doi: 10.1016/j.neuroimage.2015.03.041.

Van der Laan, L.N., de Ridder, D.T.D., Viergever, M.A., and Smeets, P. a. M. (2011). The first taste is always with the eyes: a meta-analysis on the neural correlates of processing visual food cues. Neurolmage 55, 296-303.

Walton, M.E., Rudebeck, P.H., Bannerman, D.M., and Rushworth, M.F.S. (2007). Calculating the cost of acting in frontal cortex. Ann. N. Y. Acad. Sci. 1104, 340-356. 


\section{Figure captions}

Figure 1: Trial structure. Participants were asked to rate how much they liked each of two presented food items per trial on a 1-5 Likert scale during the valuation phase. Over the choice period participants had to choose whether they prefer the first or second presented food item.

Figure 2: Behavioral results. a) Mean response frequencies ( \pm s.e.m.) and (b) mean response times to disliked (red), mildly liked (blue) and strongly liked food items (green) during the valuation phase. c) Response times ( \pm s.e.m.) over the choice period where participants had to choose in each trial one of two food alternatives. d) Associations between response times during the food valuation phase and the choice period. * $p \leq 0.05,{ }^{* *} p \leq 0.01$.

Figure 3: Results of head-surface VEP analyses. a) Interaction obtained by an electrode and time-point-wise 2x3 ANOVA with the factors of liking and choice, b) GFP waveforms to food viewing as modulated by liking and subsequent food choices as well as bar graphs visualizing the mean GFP to each food viewing condition over the time window $135-180 \mathrm{~ms} .{ }^{* *} \mathrm{p} \leq 0.01$

Figure 4: Neural source modulations by liking and subsequent food choice over the 135-180ms interval following food image onset. a) Brain areas showing an interaction of the factors Liking and Choice b) main effect of Liking. c) Main effect of Choice. * $p \leq 0.05,{ }^{* *} p \leq 0.01$. 
Trial structure

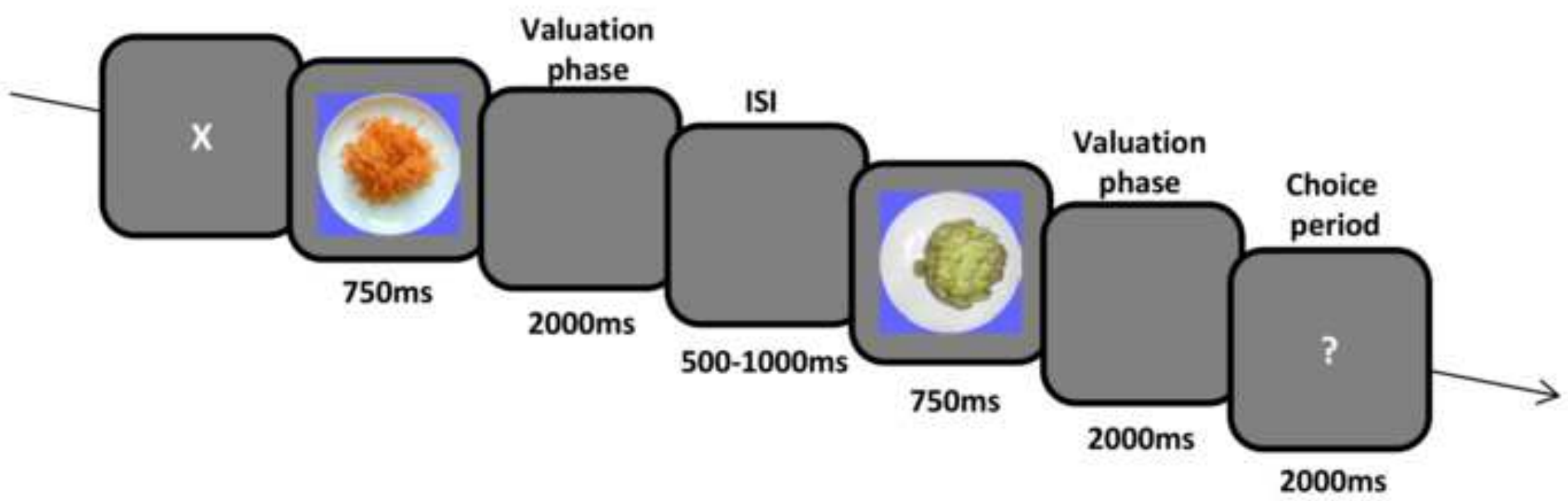


Behavioral results

a) Response frequencies during valuation phase

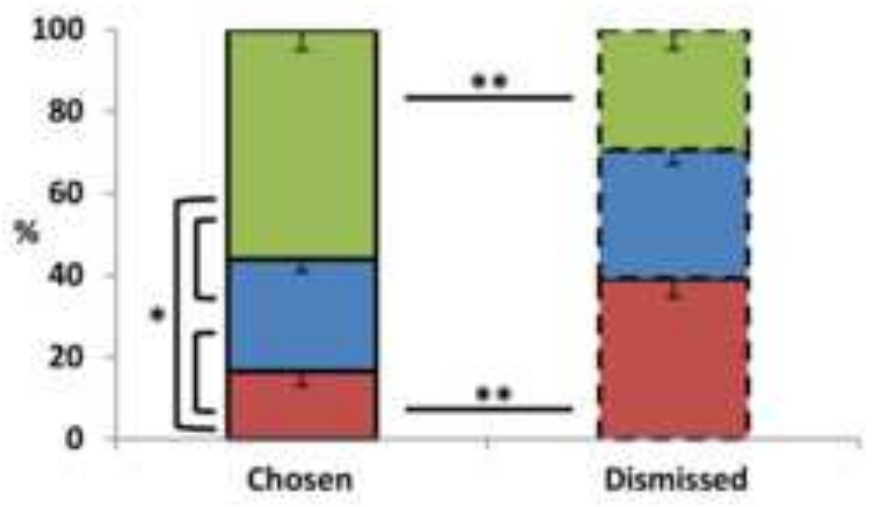

b) Response times (RTs) during valuation phase
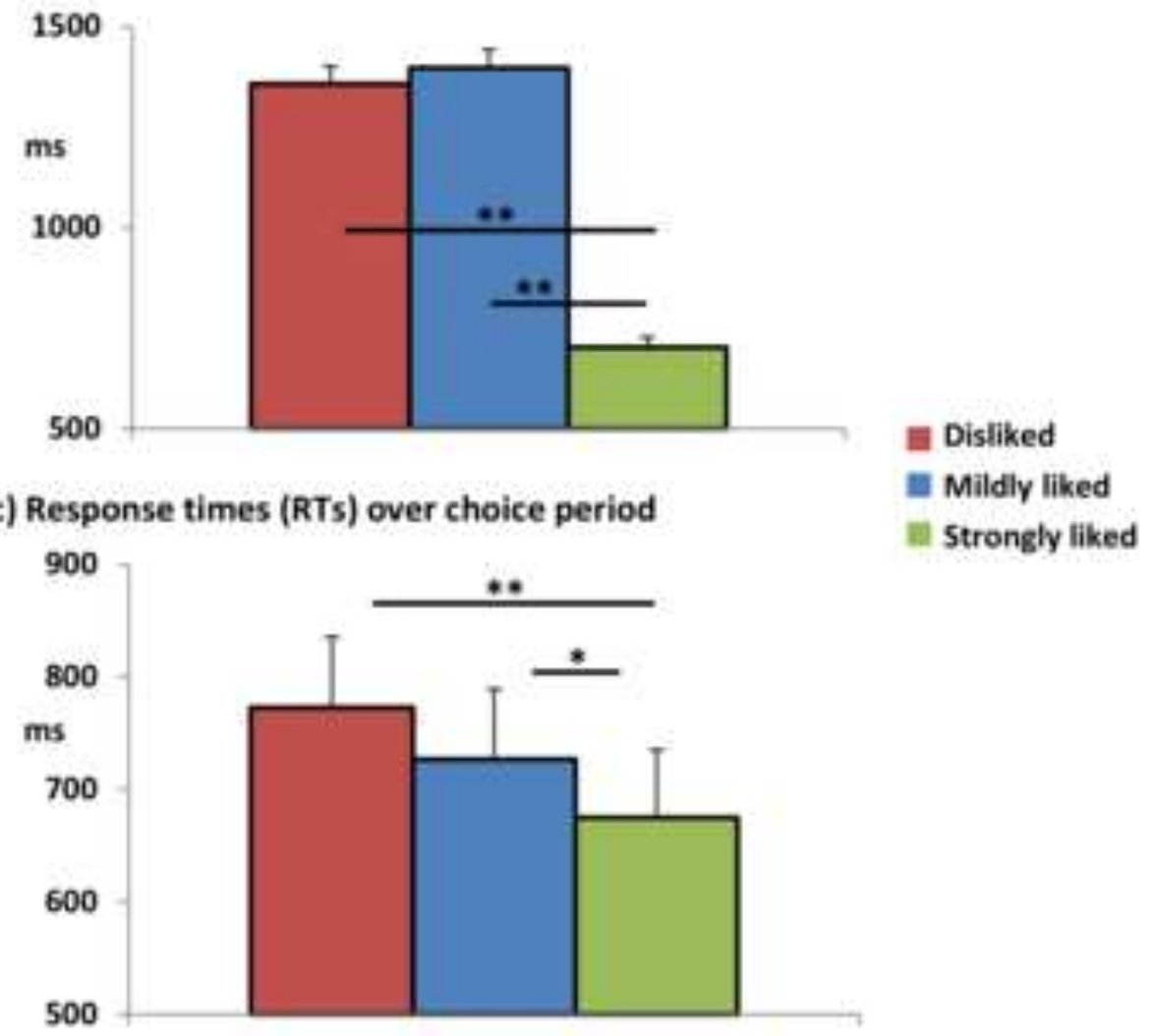

d) Associations between valuation and choice RTs: chosen foods

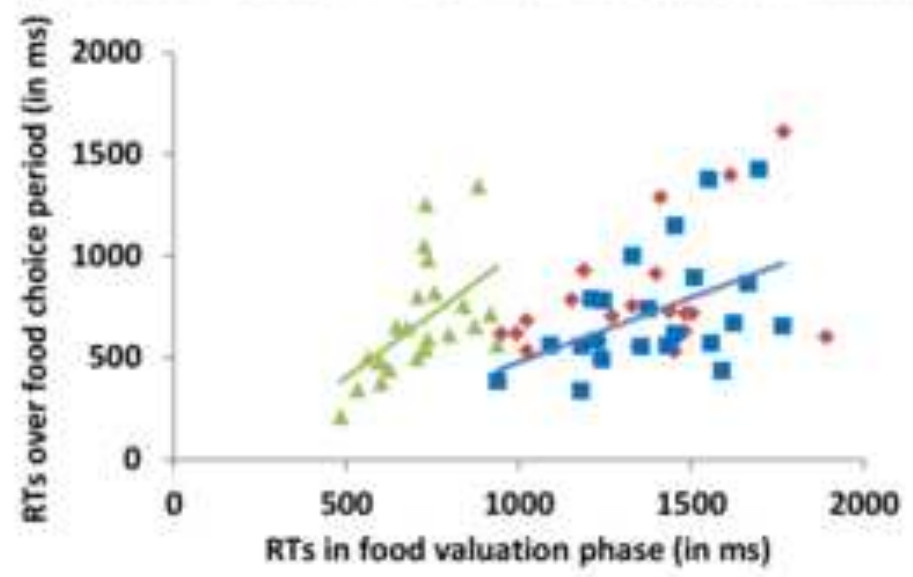


a) VEP voltage waveform results

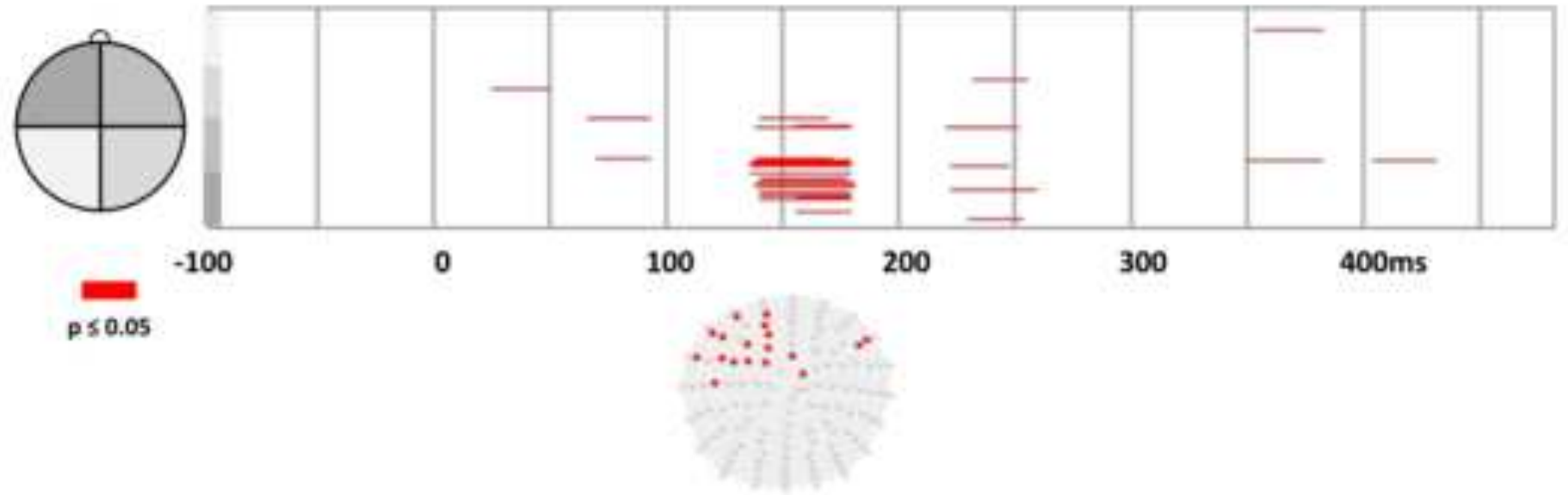

b) GFP results
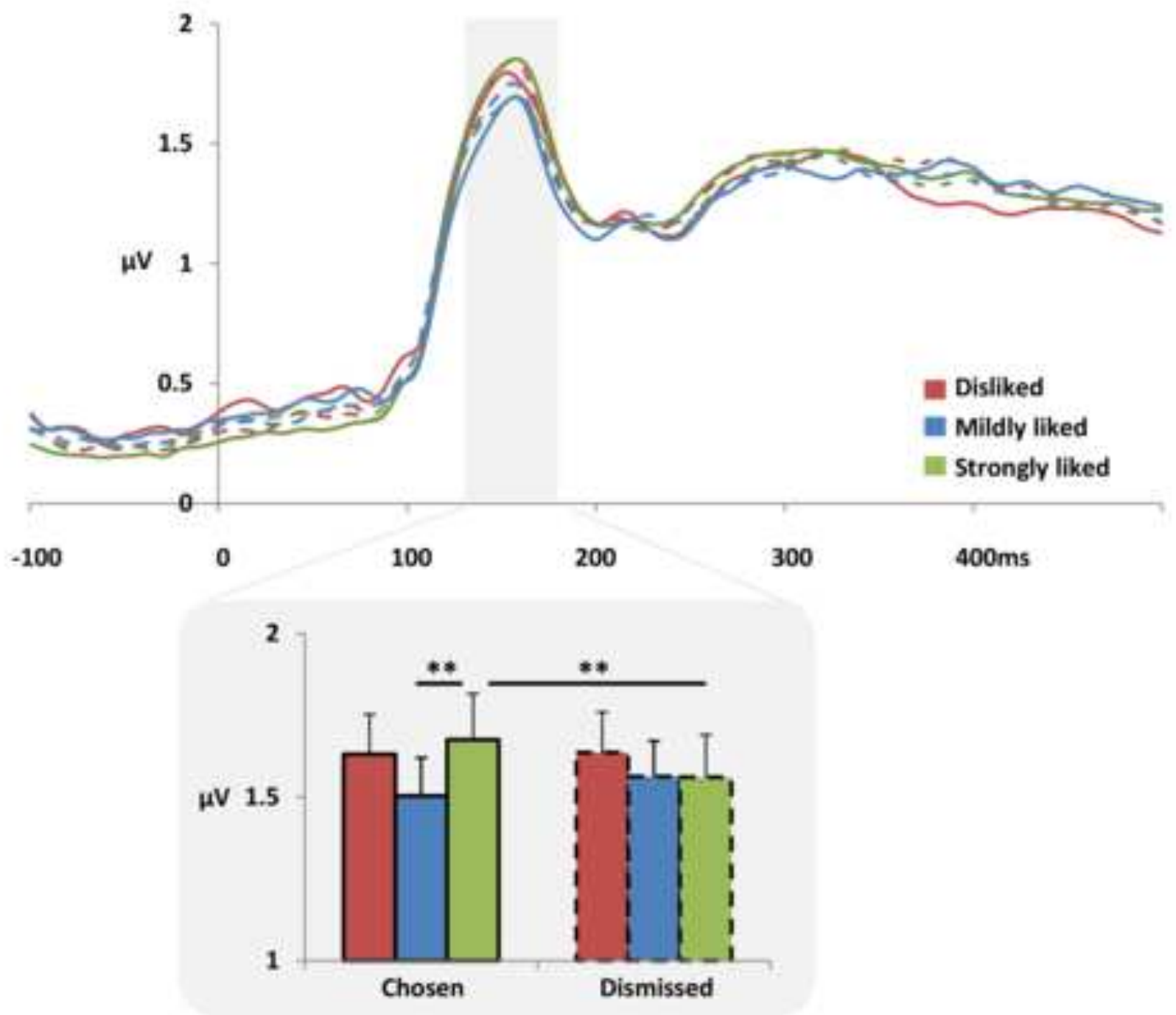
Modulations in neural source activity over the $135-180 \mathrm{~ms}$ interval following food image onset

a) Interaction Liking $x$ Choice
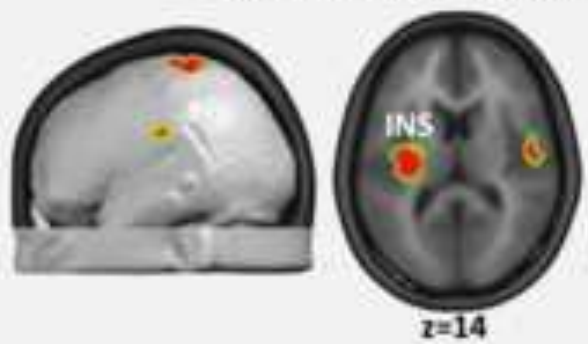

Insula

Dorsolateral (di)PFC

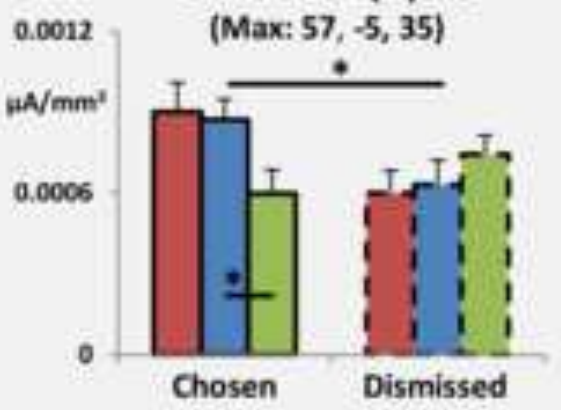

(Max: -34, -11, 14)

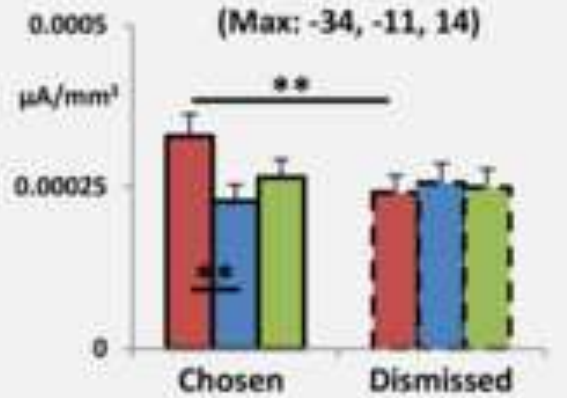

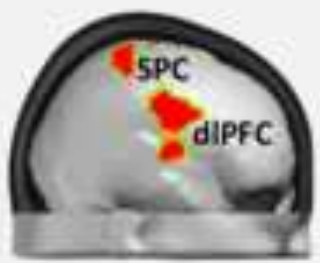

ps 0.05;

$>10$ cluster nodes

Disliked Eildly liked E Strongly liked

b) Main effect of Liking
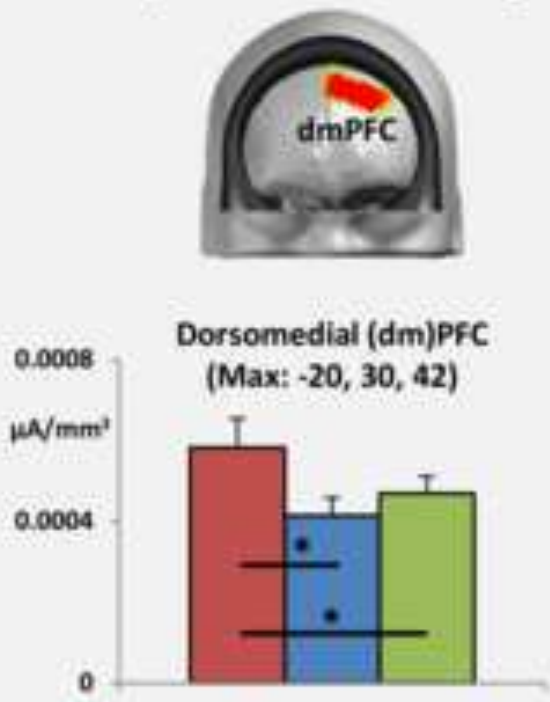

Eistiked = Mildly liked 1 Strongly liked c) Main effect of Choice
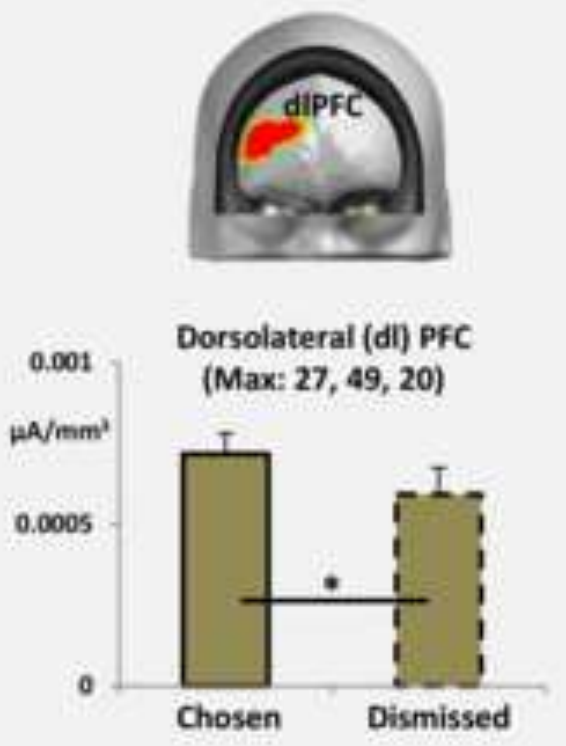